\title{
Electrical Response of $\beta$-PVDF in a Constant Uniaxial Strain Rate Deformation
}

\author{
V. SENCADAS \\ Deptartamento de Física \\ Universidade do Minho \\ 4710-057 Braga, Portugal and \\ Department of Polymer Engineering \\ University of Minho \\ 4800-058 Guimarães, Portugal

\section{MOREIRA} \\ Deptartamento de Física \\ Universidade do Minho \\ 4710-057 Braga, Portugal

\section{J. F. MANO} \\ Department of Polymer Engineering \\ University of Minho \\ 4800-058 Guimarães, Portugal and \\ 3B's Research Group \\ University of Minho \\ 4710-057 Braga, Portugal
}

\section{S. LANCEROS-MÉNDEZ}

Deptartamento de Física

Universidade do Minho

4710-057 Braga, Portugal

Received August 8, 2003; in final form January 5, 2004

The microstructure of $\beta-P V D F$ has great influence on its piezo- and pyroelectric responses. The microstructure of $\beta-P V D F$ drastically changes upon a mechanical deformation perpendicular to the preferred chain orientation, mainly above the yielding point. The voltage (open-circuit response) developed in $\beta-P V D F$ films was monitored while the material is subjected to a constant strain rate program.

Keywords Ferroelectrics; polymers; electromechanical

PACS: 62.20.Fe; 77.65.-j; 77.84.-s; 77.90.+k 


\section{Introduction}

Polyvinylidene fluoride (PVDF) in its $\beta$-phase is known for its piezo- and pyroelectric properties, offering a broad range of applications [1]. PVDF is usually found in a semicrystalline form (40-50\% crystallinity) and its microstructure has great influence on its piezo- and pyroelectric responses [2]. A mechanical deformation perpendicular to the preferred chain orientation induces realignment of the polymeric chains towards the stress field [3, 4], which influences the dielectric function of the material [4]. The effect of chain reorientation on the voltage developed in $\beta$-PVDF films is monitored while the material is subjected to a constant strain rate deformation.

\section{Experimental}

Strips of $8 \mathrm{~cm} \times 1 \mathrm{~cm}$ were cut along the two main directions (perpendicular -2- and parallel -1- to the chain orientation) from a commercial $28 \mu \mathrm{m}$ thick $\beta$-PVDF film (Measurements Specialties, Inc). The samples were electroded (area: $3 \mathrm{~cm} \times 1 \mathrm{~cm}$ ) with silver ink or by $\mathrm{Ti}$ and $\mathrm{Al}$ sputtering. Open-circuit tensile tests were carried out under a strain rate of $\mathrm{dl} / \mathrm{dt}$ $=2 \mathrm{~mm} / \mathrm{min}$ in an INSTRON 4505 apparatus at room temperature. The generated electric response was measured with an electrometer amplifier (input impedance $25 \mathrm{MOhm}$ ) and a HP3441A multimeter (input impedance $10 \mathrm{MOhm}$ ). Prior to each experiment the samples were short-circuited in order to remove the surface charges.

\section{Results}

The open-circuit voltage developed by the sample in a uniaxial strain rate deformation increases non-linearly with the applied stress and strain (Fig. 1a and b) in the pre-yielding zone and it decreases with decreasing stress and increasing deformation in the post-yielding zone. The sensitivity (charge/force) along direction 2 (Fig. 1c) shows two linear regimes in the pre-yielding zone: increases sharply for stresses up to $10 \mathrm{MPa}(\sim 2.8 \mathrm{~N})$ and with a lower slope up to the yielding point $(16.2 \mathrm{~N})$. After the yielding, a non-linear decrease occurs that accompanies the chain re-orientation process.

The electrical and mechanical hysteresis of the sample along direction 2 was measured by experiments involving the following cycle (Fig. 1d): (a) increasing stress up to a given value in the elastic region; (b) maintaining the stress constant for a given time; (c) releasing of the stress and (d) performing a stress-strain experiment up to break. A slight elastic hysteretic behaviour is translated into a more pronounced hysteresis in the voltage-deformation curve ( $\mathrm{H}$ in Fig. 1d): a remnant voltage is observed once the stress is released. Further, when the stress is applied and reaches the desired value (1 in Fig. 1d), the PVDF piezoelectric voltage continues to develop up to a maximum (2). Once the maximum voltage is achieved (2), it decays $\sim 13 \%$ over a period of $200 \mathrm{~s}$ and tends to stabilise by loosing the voltage gained at constant stress.

\section{Discussion and Conclusions}

The electromechanical performance of $\beta$-PVDF along direction 2 is lower than along direction 1 [1]. When the stress is applied parallel to the chains, a much larger, linear and reversible increase of the open-circuit voltage is observed $[1,7]$.

The electromechanical response of $\beta$-PVDF is determined by its anisotropic semicrystalline structure. So, g31 is mainly determined by a deformation of the amorphous regions, whereas g32 depends also on the deformation of the crystalline regions [5]. The piezoelectric coefficient $\mathrm{g} 32$ as a function of deformation shows a strong nonlinear behaviour and even 

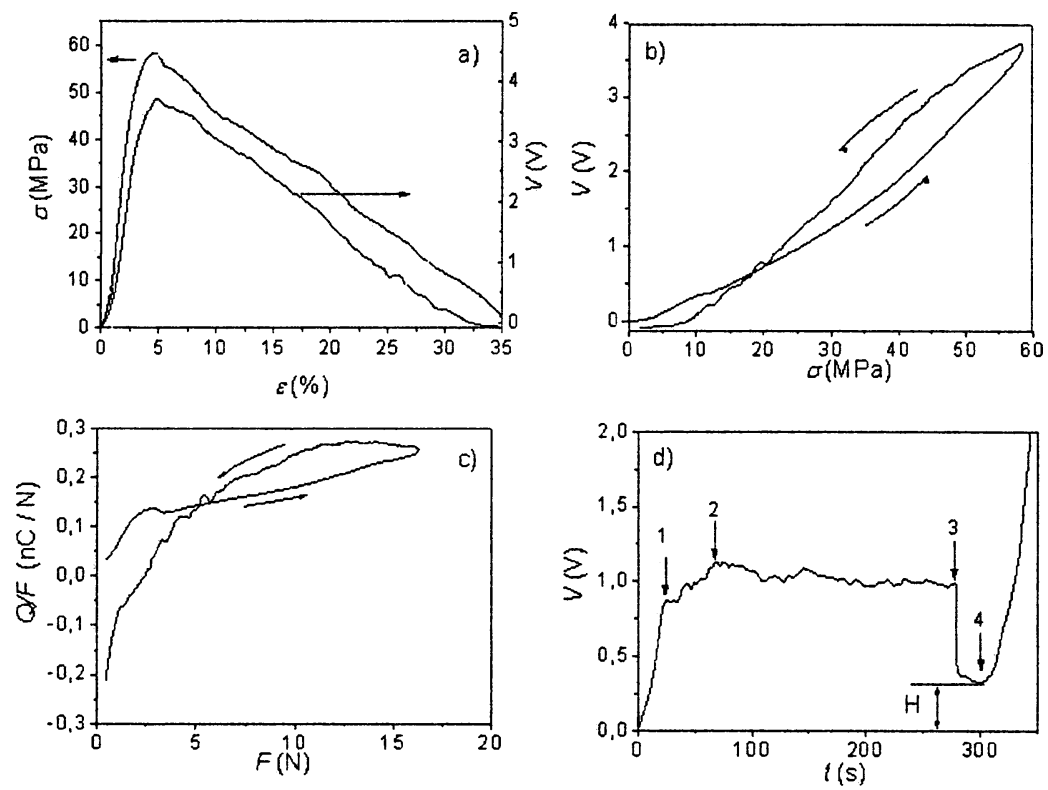

FIGURE 1 (a) Stress and voltage versus strain, (b) voltage versus stress, (c) sensitivity $(\mathrm{Q} / \mathrm{F}$ versus $\mathrm{F})$, and (d) voltage versus time $(\mathrm{dl} / \mathrm{dt}=2 \mathrm{~mm} / \mathrm{min})$ for the following stress cycle: Increasing stress up to a value of $12 \mathrm{MPa}$ from $\mathrm{t}=0 \mathrm{~s}$ to $25 \mathrm{~s}(1)$; constant stress (12 $\mathrm{MPa}$ ) up to $\mathrm{t}=275 \mathrm{~s} \mathrm{(3);} \mathrm{release} \mathrm{of} \mathrm{stress} \mathrm{(3)} \mathrm{and} \mathrm{stress-strain} \mathrm{experiment} \mathrm{starting} \mathrm{at} \mathrm{t}=$ $300 \mathrm{~s}$ (4), for $\beta$-PVDF for constant deformation experiments along direction 2.

change sign at deformations of $\sim 30 \%$, which is coincident with the complete realignment of the polymeric chains [4, 7]. For increasing stress, the g32 increases in two linear regimes (change of regime at $\sim 10 \mathrm{MPa}(\sim 2.8 \mathrm{~N})$ ) and decreases nonlinearly after the yielding point, becoming also negative, when the chain reorientation is completed [7]. The nonlinearity in the piezoelectric response can be attributed to the properties of the crystalline regions and to interface effects. The g32 behaviour can be interpreted as mechanically induced depolarization due to intrinsic effects related with changes in dipole orientation. Mechanically induced changes in the angular orientation distribution of the chain stems carrying the dipole moments have been reported $[3,4]$.

Furthermore, the increase of the voltage once the stress achieves a given value (1-2 in Fig. 1d) and its posterior decay (2-3) is explained considering a delayed process induced by finite conductivity in the two phases of PVDF [6]. The fast response component originates from the piezoelectric response from the dipoles in the ferroelectric phase, while the second slow component is controlled by conductivity related charge accumulation at the interfacial boundaries.

\section{Acknowledgements}

Work supported by the Portuguese Foundation for Science and Technology under Grant POCTI/CTM/33501/99.

\section{References}

1. P. Ueberschlag, PVDF piezoelectric polymer. Sensor Review 21, 118-125 (2001). 
2. A. J. Lovinger, Developments in crystalline polymers. In D. C. Basset ed., London: Elsevier Applied Science, Vol. 1, 1982.

3. S. Lanceros-Méndez, M. V. Moreira, J. F. Mano, V. H. Schmidt, and G. Bohannan, Dielectric behavior in an oriented $\beta$-PVDF film and chain reorientation upon transverse mechanical deformation. Ferroelectrics 273, 2393-2398 (2002).

4. R. Barbosa, V. Sencadas, J. A. Mendes, V. Moreira, J. F. Mano, and S. Lanceros-Mendez, Chain reorientation in $\beta$-PVDF films upon transverse mechanical deformation studied by SEM and dielectric relaxation. Ferroelectrics (in press).

5. B. R. Hahn, Studies of the nonlinear piezoelectric response of polyvinilidene fluoride. J. Appl. Phys. 57, 1294-1298 (1985).

6. H. von Seggern and S. N. Fedosov, Conductivity-induced polarization buildup in poly(vinilidene fluoride). Appl. Phys. Lett. 51, 2830-2832 (2002).

7. S. Lanceros-Mendez, V. Sencadas, D. Krawczyk, and J. F. Mano, to be published. 